\title{
Deformation Analysis of $128 \times 128$ Infrared Detector with Reticulated InSb Pixel Array
}

\author{
Xiaoling Zhang ${ }^{1}$, Yawei Liu ${ }^{2}$, Liwen Zhang $^{3}$ and Qingduan Meng ${ }^{3, *}$ \\ ${ }^{I}$ School of Information Engineering, Henan University of Science and Technology, Luoyang 471023, China \\ ${ }^{2}$ School of Physical Education, Henan University of Science and Technology, Luoyang 471023, China \\ ${ }^{3}$ School of Electrical Engineering, Henan University of Science and Technology, Luoyang 471023, China
}

\begin{abstract}
The reticulated InSb pixel array was successfully employed in the design of large format InSb infrared focal plane arrays (IRFPAs) detector, to remove the thermal strain accumulated in InSb IRFPAs with the thermal shock test. In order to explore the deformation rules in the InSb IRFPAs with reticulated InSb pixel array, in light of the proposed equivalent modeling, a three-dimensional modeling of InSb IRFPAs is created, and the Z-component of strain is selected to compare the displacements in the various layers of InSb IRFPAs. Analyzing results that show the top surface deformation of InSb IRFPAs originates from the thermal mismatch between the silicon readout integrated circuit (ROIC) and the indium bump array directly above. After passing through the intermediate layer and the silicon substrate, the deformation amplitude along Z-direction is reduced firstly from $0.113 \mu \mathrm{m}$ to $0.0395 \mu \mathrm{m}$, finally to $0.0042 \mu \mathrm{m}$. Here the intermediate layer is made up of the indium bump array and the reticulated InSb pixel array. These deformation data suggest that the InSb IRFPAs with reticulated InSb pixel array is superior to that designed with the underfill filled structure in the fabricating large format InSb IRFPAs.
\end{abstract}

Keywords: Focal plane arrays, Deformation, Reticulated structure, Modeling.

\section{INTRODUCTION}

Infrared focal plane arrays (IRFPAs) detectors, active in $3-5 \mu \mathrm{m}$ range, have been widely used in the high-level military equipment fields, such as the ballistic missile defense system (BMDS) [1], the infrared imaging control missile system [2], and the military remote-sensing satellite [3]. For the existing well-developed IRFPAs detectors, InSb IRFPAs have excellent uniformity in the middle wavelength range, and $\mathrm{HgCdTe}$ IRFPAs occupy a dominant position in the dual or multiband detector field [4]. Compared with $\mathrm{HgCdTe}$ IRFPAs, the production process of InSb IRFPAs has both high mature technology and excellent uniformity. For example, the highest mass production rate, exceeding $3000 \mathrm{InSb}$ sensor chip assemblies per year, is held by the Raytheon Company, which delivered the largest format InSb IRFPAs of $4 \mathrm{~K} \times 4 \mathrm{~K}$ arrays in 2009 [5]. But, for large format IRFPAs, the fracture of the InSb chip in the thermal shock test has been a major problem in mass production. Up to now, there are two successful designs to solve the fracture of InSb IRFPAs. In one of the designs of InSb IRFPAs, the underfill is filled in the gap between the InSb chip and the silicon readout integrated circuit (ROIC), and both the balanced composite structure (BCS) and the thinned silicon ROIC

\footnotetext{
*Address correspondence to this author at the School of Electrical Engineering, Henan University of Science and Technology, No.263, Kaiyuan Road, Luolong District, Luoyang 471023, China; Tel: +86-379-64231157(office), +86-13525454409 (Mobile); Fax: +86-379-64231910; E-mail: qdmengly@163.com
}

design are employed to remove the thermal mismatch existing in the layered structure [6]. In the other design of $\mathrm{InSb}$ IRFPAs, the InSb photosensitive pixels are distributed in a reticulated array, and the pixels are isolated from each other, just as schematically shown in Fig. (1) [7]. Around the indium bump array, the underfill is not filled. Both the isolated $\mathrm{InSb}$ pixel array and the isolated indium bump array are placed between the silicon substrate and the silicon ROIC. This design was successfully applied in the mass production of large format InSb IRFPAs in L-3 Cincinnati Electronics [7].

In order to understand how the thermal mismatch was removed in IRFPAs fabricated with reticulated $\mathrm{InSb}$ pixel array, basing on the equivalent modeling proposed in [8], where a small format array replaces the real large format array to achieve the 3-D modeling of InSb IRFPAs, the following questions, such as: 1) Where does the thermal strain start? 2) What is the transferring rule of the thermal strain in IRFPAs with reticulated InSb pixel array? are researched in this paper.

\section{MODELING CREATION AND PARAMETERS SE- LECTION}

The structure of InSb IRFPAs is complex, and with characteristic sizes ranging from a few micrometers to several centimeters, so in its modeling creation, a large number of elements are needed, which leads to the conclusion that stiffness matrix is too large to be solved. To overcome this problem, an equivalent. 


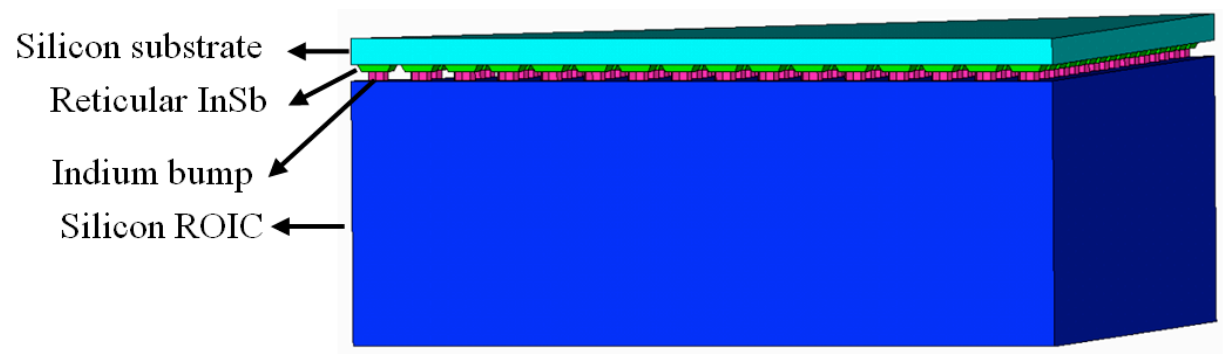

Fig. (1). Schematic of the IRFPAs with reticulated InSb pixel array.

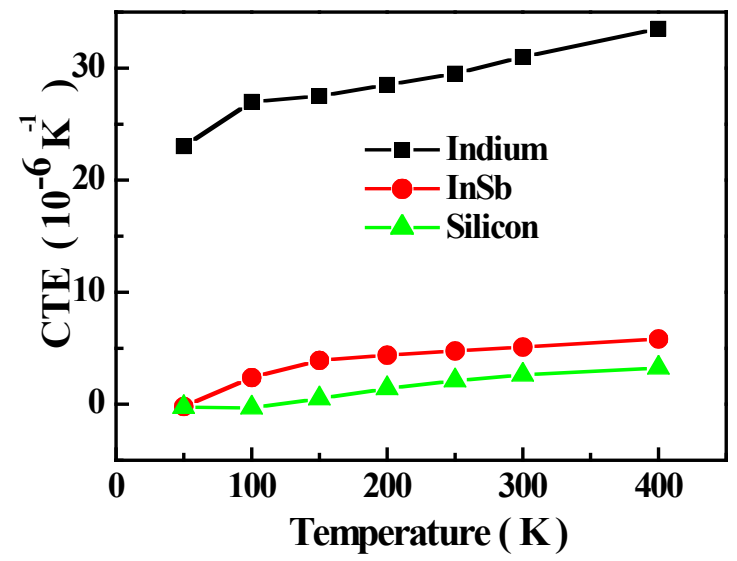

Fig. (2). Linear coefficients of thermal expansion as a function of temperature for InSb, indium and silicon.

Table I. Elastic material parameters at different temperatures.

\begin{tabular}{|c|c|c|c|}
\hline \multirow{2}{*}{ Materials } & Elastic Modulus (E/GPa) & Poison's & $\begin{array}{c}\text { Temperatures } \\
\text { Ratio }(\boldsymbol{\mu})\end{array}$ \\
\hline \hline \multirow{2}{*}{ Silicon } & 163 K) & 0.28 & $77-300$ \\
\hline \multirow{2}{*}{ InSb chip } & 409 (in plane) & 0.35 & $77-300$ \\
\cline { 2 - 4 } & 122.7 (out-of-plane) & & 76 \\
\cline { 2 - 4 } Indium bump & 20.54 & 0.4326 & 187 \\
\cline { 2 - 4 } & 16.24 & 0.4408 & 300 \\
\hline
\end{tabular}

modeling was proposed in [8], where, a smaller array is employed to replace the larger array to create the structural modeling of InSb IRFPAs. In the modeling of IRFPAs with reticulated $\mathrm{InSb}$ pixel array, the indium bump is modeled by VISCO107, both the InSb chip and the silicon material are modeled by SOLID95. Flip chip process is completed at 370 $\mathrm{K}$, at this temperature, no residual stress is assumed to exist within the whole package. In the simulation, the temperature of the InSb IRFPAs is gradually reduced from $370 \mathrm{~K}$ to 77 $\mathrm{K}$, no transient heat transfer is considered and the temperature within the model is assumed to be uniform. Symmetry boundary conditions are utilized, and displacements constrain is applied to the symmetry areas, at the same time, the bottom surface center point of the silicon ROIC also remains zero for avoiding movement. Here, the thickness of the re- ticulated $\mathrm{InSb}$ pixel is set to $8 \mu \mathrm{m}$, the diameter of indium bump is $24 \mu \mathrm{m}$ with $10 \mu \mathrm{m}$ height, the thickness of silicon ROIC is set to $300 \mu \mathrm{m}$, and the top silicon substrate is set to $30 \mu \mathrm{m}$.

The Coefficient of thermal expansion (CTE) of material is strongly dependent on the temperature, which usually decreases with decreasing temperature. In order to calculate exactly the accumulated thermal strain in the layered structure, the employed CTE models are all temperaturedependent. Silicon ROIC is a homogeneous and isotropic linear elastic material, InSb is an anisotropic linear elastic material. Indium bump is a viscoplastic material and its Young's modulus increases with decreasing temperature. The CTEs for different materials are plotted in Fig. (2) [9]. 


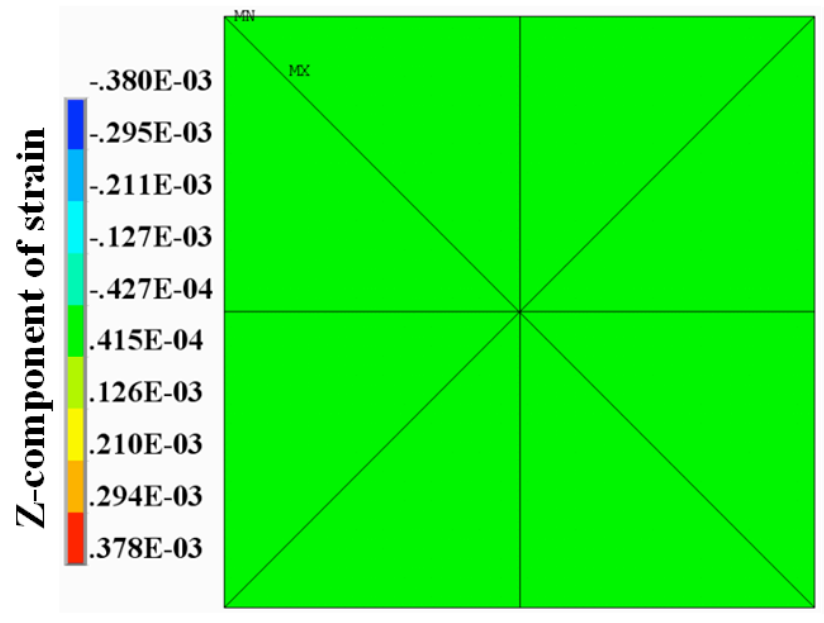

(a)

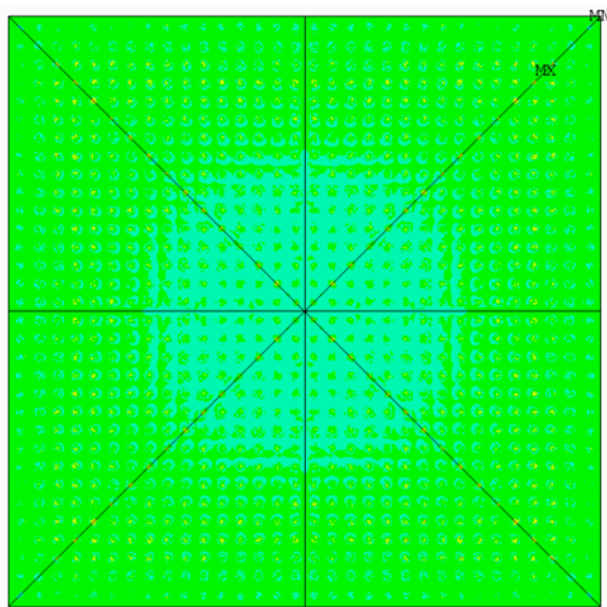

(b)

Fig. (3). Simulated Z-component of strain in the silicon ROIC: (a) bottom surface; (b) top surface.

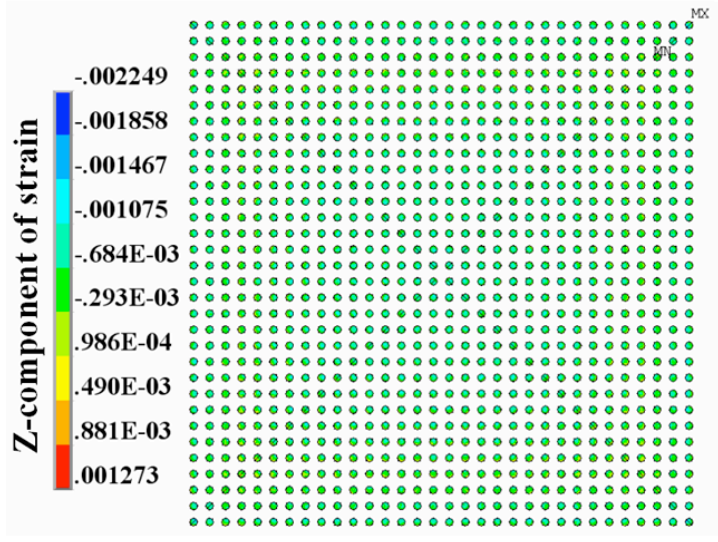

(a)

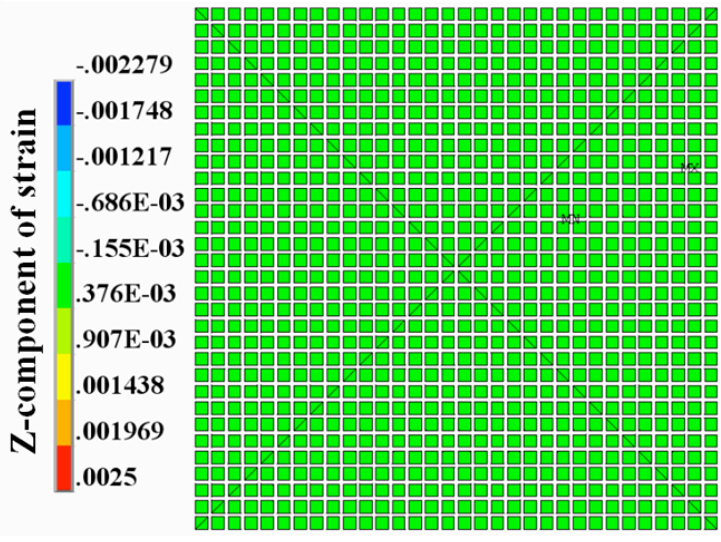

(b)

Fig. (4). Simulated Z-component of strain in the intermediate layer composed by indium bump array and reticulated InSb pixel array: (a) strain distribution on the top surface of indium bump array top surface, (b) strain distribution on the top surface of reticulated InSb pixel array.

All the employed mechanical properties parameters are listed in Table 1 [10]. Where $E$ is the Young's modulus, $\mu$ is the Poisson's ratio.

\section{SIMULATION RESULTS AND DISCUSSION}

The simulated Z-component of strain on the surface of silicon ROIC is shown in Fig. (3). Fig. (3a) is the strain on the bottom surface of silicon ROIC, Fig. (3b) is the strain on its top surface. Apparently, on the bottom surface of silicon ROIC, the strain distribution is uniform and maintains at zero, which implies the bottom surface of silicon ROIC remains flat in the thermal shock test and no distortion occurs. Yet on its top surface, the Z-component of strain in the locations contacted with the indium bump array is larger than its surrounding part, and the locations in contact with the indium bump array are convex upwards. In its top surface center, the silicon ROIC is slightly concave downwards. Taking account of the silicon ROIC with $300 \mu \mathrm{m}$, the maximal convex amplitude is derived at $0.113 \mu \mathrm{m}$.
In the IRFPAs with reticulated InSb pixel array, the InSb photosensitive pixel is one-to-one contact with the indium bump. The simulated Z-component of strain distribution in the top surface of the indium bump array and the reticulated InSb pixel array are shown in Fig. (4a and $\mathbf{b}$ ), respectively. Although, on the top surface of silicon ROIC, its center part is concave downwards, and its edge part is convex upwards, the uneven top surface of silicon ROIC becomes even and smooth after passing through the indium bump array and the reticulated InSb pixel array. On the whole top surface of indium bump array and the reticulated $\mathrm{InSb}$ pixel array, without any slopes and hills. The maximal Z-component displacements are $0.0127 \mu \mathrm{m}$ and $0.0200 \mu \mathrm{m}$, respectively. On the whole top surface of the reticular InSb photosensitive pixel arrays, the amplitude of the rise and fall along Zdirection is smaller than $0.0382 \mu \mathrm{m}$.

The Z-component of strain on the surface of silicon substrate is shown in Fig. (5). In its center part, the silicon substrate is convex upwards, and at its four corners, the silicon 


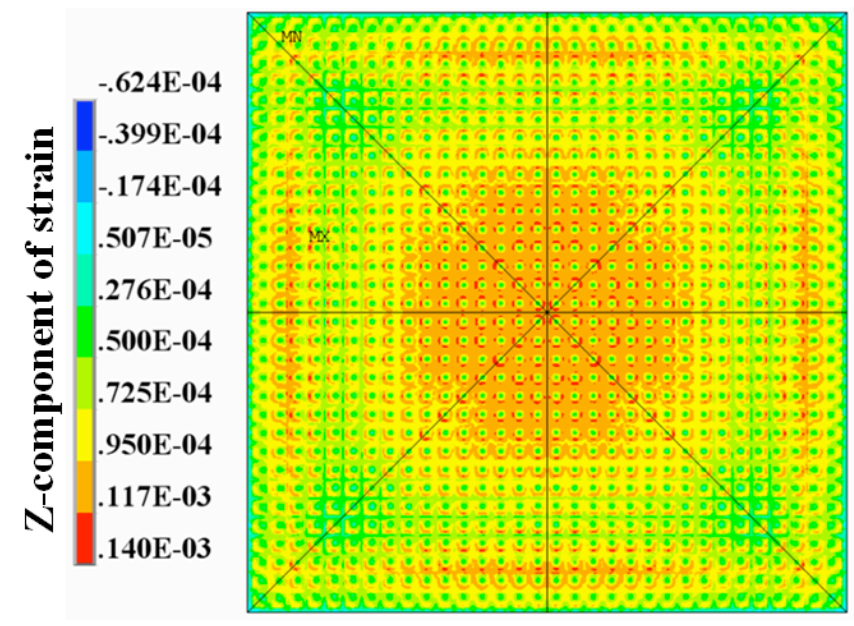

(a)

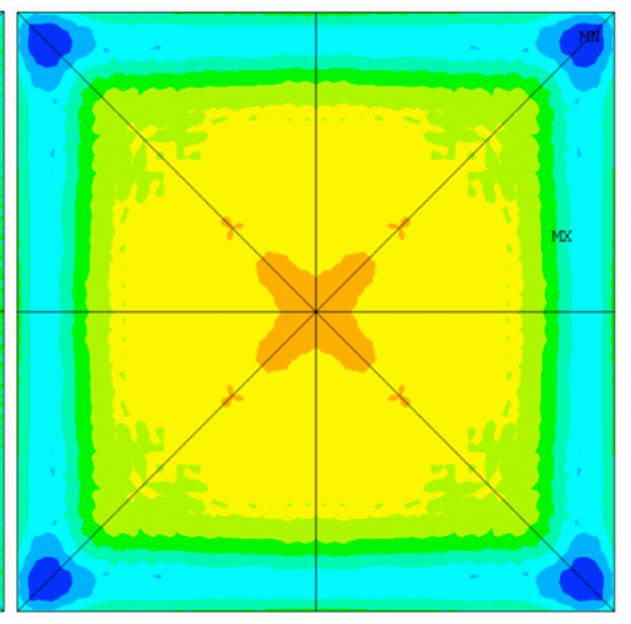

(b)

Fig. (5). Simulated Z-component of strain in the silicon substrate: (a) bottom surface; (b) top surface.

substrate is concave downwards. The deformation distribution along Z-direction in the silicon substrate is contrary to the deformation distribution appearing on the top surface of silicon ROIC, where the silicon ROIC is slightly concave downwards in its top surface center. Furthermore, we notice that on the bottom surface of silicon substrate, the Zcomponent of strain in the silicon substrate touching with InSb pixels is smaller than its surrounding part, at which the silicon substrate does not touch with InSb pixels. This is also contrary to the Z-component of strain distribution on the top surface of silicon ROIC, where, the Z-component of strain in the silicon ROIC touching with the indium bump array is larger than its surrounding part. Taking account of the silicon substrate with $30 \mu \mathrm{m}$, the maximal deformation in the positive $\mathrm{Z}$-direction is derived at about $0.0042 \mu \mathrm{m}$, and the maximal deformation in the negative Z-direction is derived at about $0.0019 \mu \mathrm{m}$.

In our previous research, we noticed that the square checkerboard buckling pattern usually appears on the back surface of InSb IRFPAs, in which the underfill is filled in the gap between the InSb chip and the silicon ROIC. In the typical deformation photograph of InSb IRFPAs, the InSb chip touched with the indium bump array shifts upward by 0.042 $\mu \mathrm{m}$, at the same time, the InSb chip touched with the meshy underfill shifts downward by $0.049 \mu \mathrm{m}$ [11]. In the IRFPAs with reticulated InSb pixel array, the deformation amplitude in Z-direction is reduced by about $90 \%$. All these simulation results prove that the IRFPAs fabricated with reticulated $\mathrm{InSb}$ pixel array is more suitable in fabricating large format IRFPAs.

In order to show clearly the transferring rule of the thermal strain in the IRFPAs with reticulated InSb pixel array, the maximal deformation in the different layers along Zdirection is plotted in Fig. (6), here, the sum deformation of the indium bump array and the reticulated InSb pixel array is also provided owing to their identical isolated feature. Apparently, the deformation in the IRFPAs with reticulated InSb pixel array along Z-direction originated from the interaction between the silicon ROIC and the indium bump array layer directly above, after passing through the $18 \mu \mathrm{m}$ inter- mediate layer, which is composed of the indium bump array and the InSb pixel array, the deformation amplitude is reduced from $0.113 \mu \mathrm{m}$ to $0.0395 \mu \mathrm{m}$. Afterwards, passing upward through the $30 \mu \mathrm{m}$ silicon substrate, the maximal deformation is further decreased to $0.0042 \mu \mathrm{m}$. In the indium bump array and the InSb pixel array, the maximal deformation along Z-direction is $0.0127 \mu \mathrm{m}$ and $0.0200 \mu \mathrm{m}$, respectively. Here the deformation amplitude in the indium bump array is smaller than that occurring in the InSb pixel array. This phenomenon originates from the fact that the indium bump is described by viscoplastic element type in the modeling.

\section{CONCLUSION}

In light of the proposed equivalent modeling, a threedimensional modeling of InSb IRFPAs with reticulated InSb pixel array is created. Analyzing results show that the deformation rules in InSb IRFPAs with reticulated InSb pixel array is almost identical with that in InSb IRFPAs filled with underfill. But the deformation amplitude in InSb IRFPAs

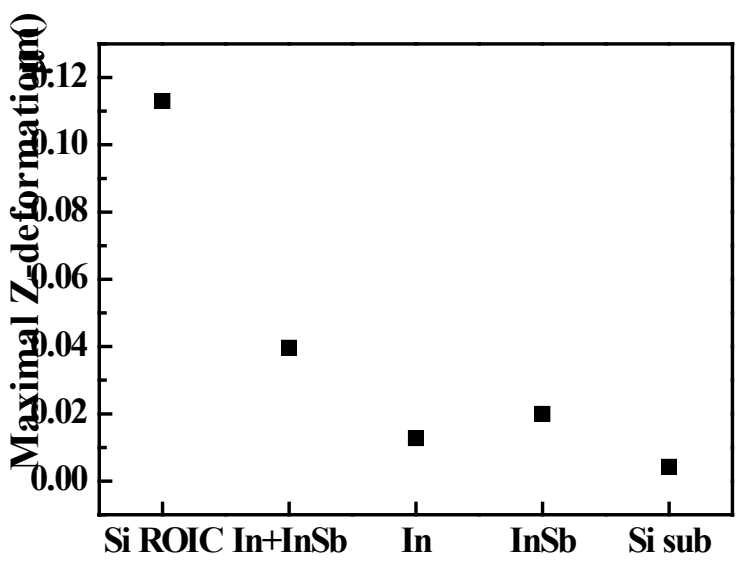

Fig. (6). Maximal Z-direction deformation in the different layers of InSb IRFPAs. 
with reticulated $\mathrm{InSb}$ pixel array is much smaller than that in InSb IRFPAs with underfill filled structure. These findings suggest that the InSb IRFPAs with reticulated InSb pixel array is more superior in designing large format InSb IRFPAs.

\section{CONFLICT OF INTEREST}

The authors confirm that this article content has no conflicts of interest.

\section{ACKNOWLEDGEMENTS}

The financial support of the Young Scientists Fund of the National Natural Science Foundation of China under Grant 61107083 and Grant 61205090 are also greatly appreciated.

\section{REFERENCES}

[1] M.Z. Tidrow, "New infrared sensors for ballistic missile defense", Proc. SPIE 5732, pp. 217-224, 2005.
[2] Available at: http://www.raytheon.com.au/rtnwcm/groups/rau/documents/download/raufactsheet aim-9x.pdf.

[3] H.M. Gong and D.F. Liu, "Developments and trends in space borne infrared detectors", Infrared Laser Eng. vol. 37, pp. 18-24, 2008.

[4] Rogalski, "Recent progress in infrared detector technologies", Infrared Phys. Technol. vol. 54, pp. 136-154, 2011.

[5] A.W. Hoffman, E. Corrales, P.J. Love, and J. Rosbeck, M. Merrill, A. Fowler, and C. McMurtry, " $2 \mathrm{~K} \times 2 \mathrm{~K}$ InSb for astronomy", Proc. SPIE 5499, pp. 59-67, 2004.

[6] P.J. Love, A.W. Hoffman, D.J. Gulbransen, M.P. Murray, K.J. Ando, N.J. Therrien, J.P. Rosbeck, and R.S. Holcombe, "Largeformat $0.85-2.5$ micron $\mathrm{HgCdTe}$ detector arrays for lowbackground applications", Proc. SPIE 5167, pp. 134-143, 2004.

[7] M. Davis and M. Greiner, "Indium antimonide large-format detector arrays", Opt. Eng., vol. 50, no. 6, pp. 1-6, 2011.

[8] Q.D. Meng, X.L. Zhang, L.W. Zhang, and Y.Q. Lv, "Structural modeling of $128 \times 128 \mathrm{InSb}$ focal plane array detector $128 \times 128$ InSb", Acta Phys. Sin. vol. 61, no. 19, 1-6, 2012.

[9] R.W. Chang and M.F. Patrick, "Constitutive relations of indium in extreme temperature electronic packaging based on Anand model", J. Electron. Mater. vol. 38, no. 9, pp. 1855-1859, 2009.

[10] Q.D. Meng, Q. Yu, L.W. Zhang, and Y.Q. Lv, "Mechanical parameters selection in InSb focal plane array detector normal direction", Acta Phys. Sin. vol. 61, no. 22, pp. 1-5, 2012.

[11] X.L. Zhang, Q.D. Meng, Q. Yu, L.W. Zhang, and Y.Q. Lv, "Thermal buckling analysis in InSb focal plane array detector", J. Mech. Sci. Technol., vol. 27, no. 6, pp. 1809-1813, 2013.

Received: June 09, 2014

Revised: June 22,2014

Accepted: July 24, 2014

(C) Zhang et al.; Licensee Bentham Open.

This is an open access article licensed under the terms of the Creative Commons Attribution Non-Commercial License (http://creativecommons.org/licenses/ by-nc/4.0/) which permits unrestricted, non-commercial use, distribution and reproduction in any medium, provided the work is properly cited. 